\title{
Low-frequency absorption using a two-layer system with active control of input impedance
}

\author{
Pedro Cobo $^{\mathrm{a})}$ and Alejandro Fernández \\ Instituto de Acústica, CSIC. Serrano 144, 28006 Madrid, Spain \\ Olivier Doutres \\ ENSIM, Université du Maine, Rue Aristote, 72085 Le Mans, France
}

(Received 25 February 2003; revised 24 September 2003; accepted 3 October 2003)

\begin{abstract}
Broadband noise absorption, including low frequencies, may be obtained by a hybrid passive-active two-layer system. A porous layer in front of an air layer provides passive absorption, at medium and high frequencies. Active control of the input impedance of the two-layer system yields absorption at low frequencies. The active control system can implement either pressure-release or impedance-matching conditions. A simple analytical model based upon plane waves propagating in a tube permits the comparison of both control strategies. The results of this simple model show that the pressure-release condition affords higher absorption than the impedance-matching condition for some combinations of geometrical and material parameters. Experimental results corroborate the good performance of the pressure-release condition under the prescribed geometrical setup. (C) 2003 Acoustical Society of America. [DOI: 10.1121/1.1629306]
\end{abstract}

PACS numbers: 43.55.Ev, 43.50.Ki [KAC]

Pages: $3211-3216$

\section{INTRODUCTION}

The demand for noise reduction, especially at low frequencies, motivates investigation using active control. Passive control provides noise reduction at medium and high frequencies with moderate performance-to-cost ratio, whereas active control is capable of canceling low-frequency noise. Therefore, active control is a complementary, rather than alternative, technique to passive control of noise. A broadband noise problem requires a hybrid passive-active noise control solution.

Noise in halls is an example that requires a broadband solution. Absorbers are typically used to decrease reverberation, this in turn reduces the reflected component of the acoustic field in rooms. Manufacturers provide materials with moderate thicknesses that have absorption coefficients close to 1 for frequencies above $1 \mathrm{kHz}$. The first peak in the frequency response of the absorption coefficient can be moved to lower frequencies by including an air gap between the absorbing layer and the wall. Increasing the thickness of the air layer will decrease the frequency of the first peak. Thus, this offers an option of designing fully passive lowfrequency absorbers. This technique, however, has two severe shortcomings: (1) the two-layer absorber can be too bulky; and (2) the first minimum of the absorption frequency response is moved towards frequencies where higher absorption is required. A solution to this problem is to design the two-layer system to yield high absorption above a few hundred hertz and complement the low-frequency range with an active system. Lueg already proposed to construct walls as vibrated membranes to silence rooms in 1933 (as discussed by Guicking, 1990).

In an active system, the controller drives the actuator with a signal synthesized from the disturbance signal accord-

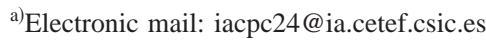

ing to some performance objective and control strategy (Elliott, 2001). Previous papers have discussed active control of absorption with different disturbance signals and control strategies. Guicking et al. (1985) applied adjustable electronic to afford active impedance or reflection control at the end of a standing wave tube. A standing wave separator calculated the disturbance signal (the incident wave) using measurements from two microphones spaced along the tube, which was processed by the controller. The controller yielded purely active absorption below $1000 \mathrm{~Hz}$. OrduñaBustamante and Nelson (1992) also used a two-microphone system to calculate a disturbance signal using a feedforward active control system for impedance matching in front of the actuator. Furstoss et al. (1997) demonstrated the superior performance of the active absorption system when this is combined with the passive absorption yielded by a layer of absorbing material spaced in front of the actuator. A microphone in the air layer provided the disturbance signal for both feedback and feedforward controllers. Minimization of this disturbance signal (pressure-release condition) gave absorption coefficients above 0.9 at frequencies between 200 and $900 \mathrm{~Hz}$.

Beyene and Burdisso (1997) carried out an experimental comparison between two control conditions on a two-layer hybrid passive-active absorbing system: (1) pressure release, which drove the pressure in the air layer to zero, and (2) impedance matching, which canceled the upstream wave in the air layer. To implement the impedance-matching condition a wave deconvolution circuit was used, similar to the standing wave separator used by Guicking et al. (1985), together with two microphones in the air gap. The experimental results of Beyene and Burdisso, further corroborated by Smith et al. (1999), showed the superiority of the impedance-matching condition as compared to the pressurerelease condition on the hybrid passive-active absorption for broadband noise. Nevertheless, the authors recognize that a 


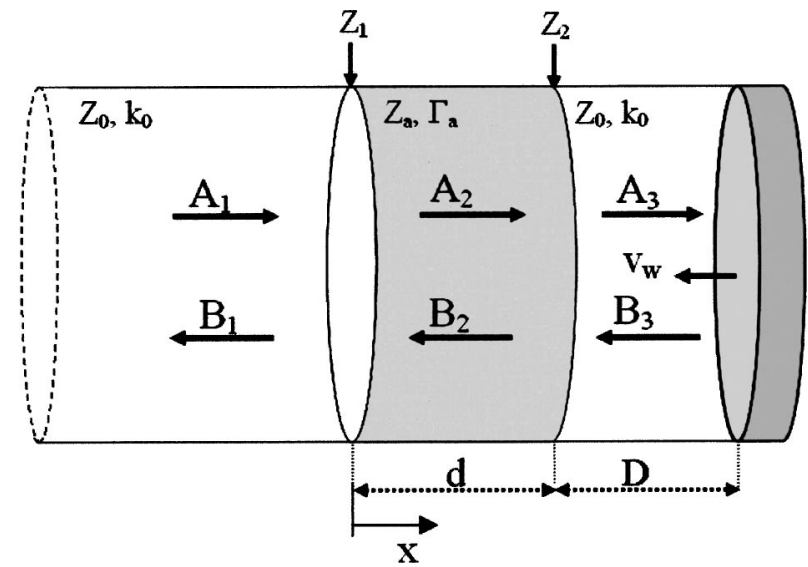

FIG. 1. Schematic representation of the hybrid two-layer passive-active system.

confirmation of this experimental result would require an analytical model that includes the physical properties of the absorption material. The main goal of this paper is to derive such an analytical model. A simple problem of plane waves propagating in a tube is considered. The passive-active absorbing system consists of a layer of porous material separated from an active wall by an air cavity. The semiempirical equations of Allard and Champoux (1992), instead of those of Delany and Bazley (1970), are used to model the propagation through the absorbing material due to the lowfrequency contents of the noise. The model yields the absorption coefficient of the system as a function of the geometrical parameters of the system (thicknesses of porous and air layers), a constitutive parameter of the absorbing material (flow resistivity), and the frequency. Following Mechel (1988), expressing these parameters in nondimensional variables offers a clearer comparison of results. When results are presented in such a way it is evident that the pressure-release condition can yield an absorption coefficient higher than the impedance-matching condition for certain combinations of parameters. Experimental confirmation is reported.

\section{ONE-DIMENSIONAL PLANE-WAVE MODEL}

Figure 1 shows a schematic representation of a hybrid passive-active two-layer system. A plane wave propagates downstream in a one-dimensional medium (a tube, for instance) from a source somewhere on the left. The absorption system at the end of the tube consists of two layers in front of an active wall that moves as a piston. The first layer, of thickness $d$, is made of a porous material. An air layer, of thickness $D$, exists between the porous layer and the active wall. Let $Z_{0}=\rho_{0} c_{0}$ and $k_{o}$ be the characteristic acoustic impedance and acoustic wave number of the air, respectively. The porous layer is characterized by a complex acoustic impedance $Z_{a}$, and a complex propagation constant $\Gamma_{a}=\alpha_{a}$ $+i k_{a}$, where $\alpha_{a}$ is the attenuation constant and $k_{a}$ is the phase constant. After reflection and transmission at the interfaces $x=0, x=d$, and $x=d+D$, downstream and upstream plane-wave fields are generated. The downstream and upstream waves have pressure amplitudes $A_{n}$ and $B_{n}, n=1,2$, and 3, respectively, as shown in Fig. 1. The absorption coefficient at the input of this system is

$$
\alpha=1-|r|^{2},
$$

where

$$
r=\frac{z-1}{z+1}
$$

is the reflection coefficient, and

$$
z=\frac{Z_{1}}{Z_{0}}
$$

is the input impedance of the system normalized to that of the air. The input impedance of this two-layer system is (Beyene and Burdisso, 1997)

$$
Z_{1}=Z_{a} \frac{Z_{2} \cosh \left(\Gamma_{a} d\right)+Z_{a} \sinh \left(\Gamma_{a} d\right)}{Z_{a} \cosh \left(\Gamma_{a} d\right)+Z_{2} \sinh \left(\Gamma_{a} d\right)},
$$

where $Z_{2}$, the input impedance to the air layer, assuming a time dependence $e^{i \omega t}$, is (Beyene and Burdisso, 1997)

$$
Z_{2}=Z_{0} \frac{V_{w} Z_{0}+2 B_{3} e^{i k_{0} d} \cos \left(k_{0} D\right)}{V_{w} Z_{0}+2 i B_{3} e^{i k_{0} d} \sin \left(k_{0} D\right)} .
$$

$V_{w}$ is the particle velocity at the active termination. Equation (5) reduces to $Z_{2}=-i Z_{0} \cot \left(k_{0} D\right)$ when $V_{w}=0$, the input impedance of the air layer for a rigid termination at $x=d$ $+D$, in the following called the passive case (Mechel, 1988).

In order to complete the propagation model for this twolayer system, the acoustical properties of the fibrous layer must be specified. Empirical and analytical models exist for propagation through such materials. For the purpose of this work, an empirical model affords enough precision and will be adopted. Most of the empirical models of propagation in absorbing materials give the acoustic impedance, $Z_{a}$, and the propagation constant, $\Gamma_{a}$, as functions of the nondimensional parameter $E=\rho_{0} f / R_{1}$, where $\rho_{0}$ is the density of the air, $f$ is the frequency, and $R_{1}$ is the flow resistivity of the material. Much of the literature on passive absorbers refers to the work of Delany and Bazley (1970). Nevertheless, these equations are restricted to the range $0.01<E<1$, which is insufficient for the low-frequency contents of the noise involved in active control. The empirical equations of Allard and Champoux (1992) can be used for any $E<1$ providing results similar to those of Delany and Bazley in the valid frequency range. Therefore, the absorbing layer will be characterized by the equations of Allard and Champoux (1992) given by

$$
\begin{aligned}
& \Gamma_{a}=i 2 \pi f \sqrt{\rho(f) / K(f)}, \\
& Z_{\alpha}=\sqrt{\rho(f) K(f)},
\end{aligned}
$$

where

$$
\begin{aligned}
& \rho(f)=1.2+\left[-0.0364 E^{-2}-i 0.1144 E^{-1}\right]^{1 / 2}, \\
& K(f)=101320 \frac{i 29.64+\left[2.82 E^{-2}+i 24.9 E^{-1}\right]^{1 / 2}}{i 21.17+\left[2.82 E^{-2}+i 24.9 E^{-1}\right]^{1 / 2}} .
\end{aligned}
$$

Equations (1)-(7) allow the absorption coefficient of the hybrid passive-active system to be obtained as a function of geometrical parameters (the thicknesses of the two layers), material parameters (the flow resistivity), acoustical param- 


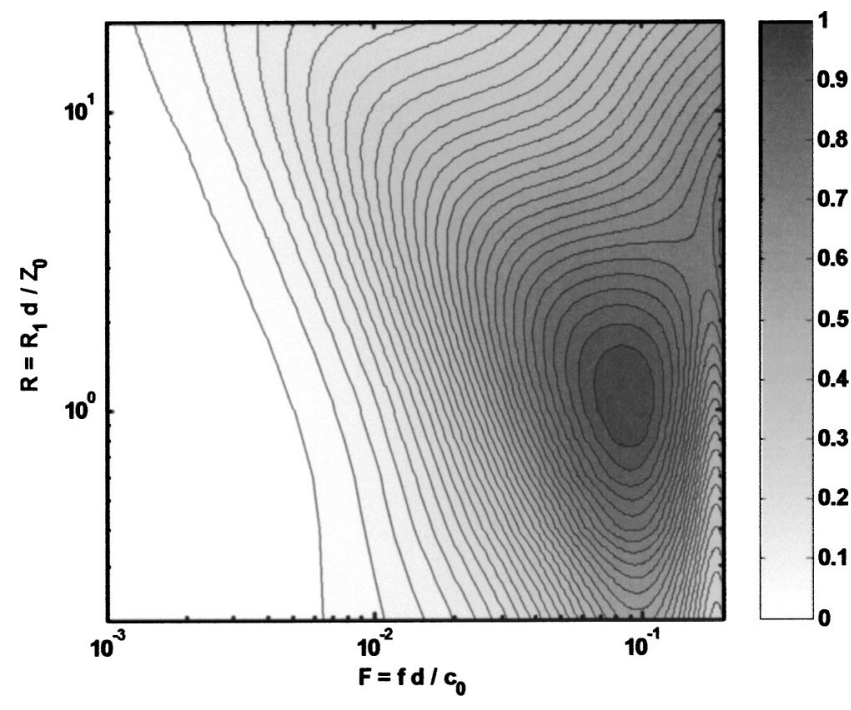

FIG. 2. Contour plot of constant passive absorption against nondimensional variables $R$ and $F$, for a two-layer system with $D=2 d$.

eters (the frequency), and the control condition $\left[V_{w}\right.$ in Eq. (5)]. Mechel (1988) showed that a better understanding of the system can be obtained when the absorption coefficient is plotted as a contour map of the nondimensional variables $F$ (defined as $f d / c_{0}$ ) and $R$ (defined as $R_{1} d / Z_{0}$ ), where $c_{0}$ is the sound speed in air. Such contour maps will be used in the following to plot the results of the model.

Concerning the control condition, several error sensors have been used, both in front of the absorbing layer and in the air layer. Beyene and Burdisso (1997) and Smith et al. (1999) carried out an extensive experimental comparison of two control conditions with the error sensor in the air layer: pressure-release and impedance-matching. In the first case, an active controller drives the total acoustic pressure in the air cavity, measured by a single microphone at the interface between the porous layer and the air cavity, to zero. This implies a zero input impedance of the air layer, $Z_{2}=0$. In the second condition, the active wall might alter the input impedance to the two-layer system, $Z_{1}$, to match that of the air, $Z_{0}$. Beyene and Burdisso (1997) demonstrate that when the acoustic thickness of the absorbing layer, $\Gamma_{a} d$, tends to zero, this condition can be achieved by driving the reflected wave to zero in the air layer, $B_{3}=0$. This happens in most absorbing materials in the low-frequency range. From Eq. (5), this condition implies that $Z_{2}=Z_{0}$. A wave deconvolution circuit converted the signals measured by two closely spaced microphones into separate incident and reflected waves in the air layer.

Figures 2-4 show contour maps of the absorption coefficient of a two-layer system with $D=2 d$, for the passive $\left(\alpha_{p}\right)$, pressure-release $\left(\alpha_{\mathrm{pr}}\right)$, and impedance-matching $\left(\alpha_{\mathrm{im}}\right)$ conditions, respectively. Ranges for the nondimensional variables $F$ and $R$ were chosen to meet the validity margin of Allard and Champoux, $E=F / R<1$. The control of the input impedance enhances the low frequency absorption for both active conditions. In the pressure-release case, the contour lines are parallel to the the $F$ axis, for $F<0.05$, with maximum absorption concentrated around $R=1$. When the acoustic pressure in the air layer vanishes, the input impedance

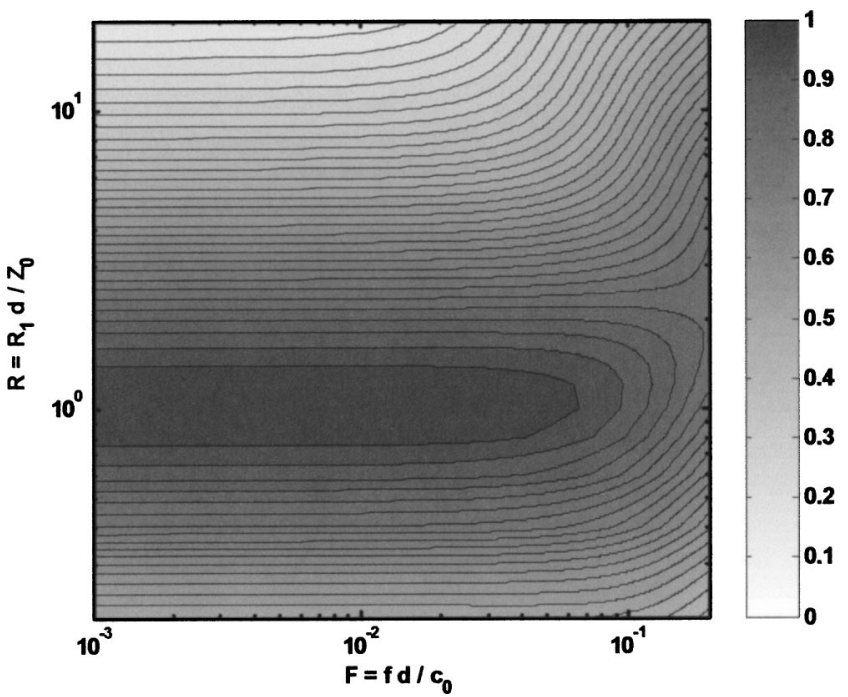

FIG. 3. Contour plot of constant passive-active absorption against nondimensional variables $R$ and $F$, for a two-layer system with $D=2 d$ using a pressure-release condition.

seen by the incident wave can be approximated by the flow resistance, $R_{1} d$ (Furstoss et al., 1997). Therefore, maximum absorption can be expected when this flow resistance matches the acoustic impedance of air, as seen in Fig. 3. The impedance-matching condition, approximated as $B_{3}=0$ in Eq. (5), requires that the argument of the hyperbolic functions in Eq. (4), $\Gamma_{a} d$, tends to zero (Beyene and Burdisso, 1997). The thinner the porous layer and the lower the flow resistivity, the closer the approximation. Thus, active control by impedance-matching condition performs better for lower $R$, Fig. 4. For a better comparison between both conditions, Fig. 5 shows contour maps of the difference of absorption coefficients, $\alpha_{\mathrm{pr}}-\alpha_{\mathrm{im}}$. The solid line represents the zerovalue contour $\left(\alpha_{\mathrm{pr}}=\alpha_{\mathrm{im}}\right)$. Above and to the left of this line, $\alpha_{\text {pr }}$ exceeds $\alpha_{\text {im }}$, and vice versa, below and right to this line, $\alpha_{\text {im }}$ exceeds $\alpha_{\mathrm{pr}}$. The value ( $\alpha_{\mathrm{im}}-\alpha_{\mathrm{pr}}$ ) for lower $R$ is much higher than the value $\left(\alpha_{\mathrm{pr}}-\alpha_{\mathrm{im}}\right)$ for medium $R$.

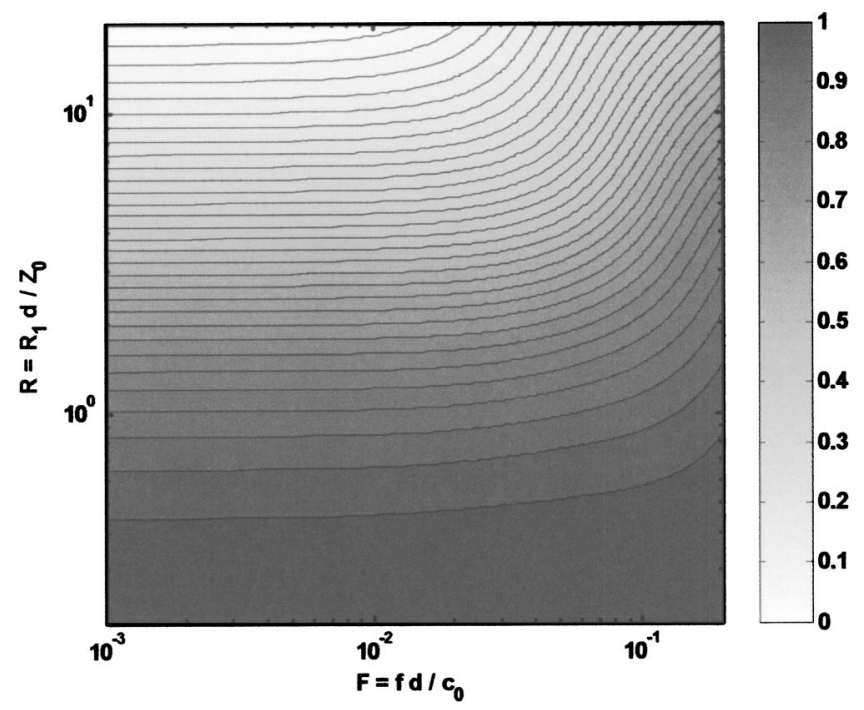

FIG. 4. Contour plot of constant passive-active absorption against nondimensional variables $R$ and $F$, for a two-layer system with $D=2 d$ using an impedance matching condition. 


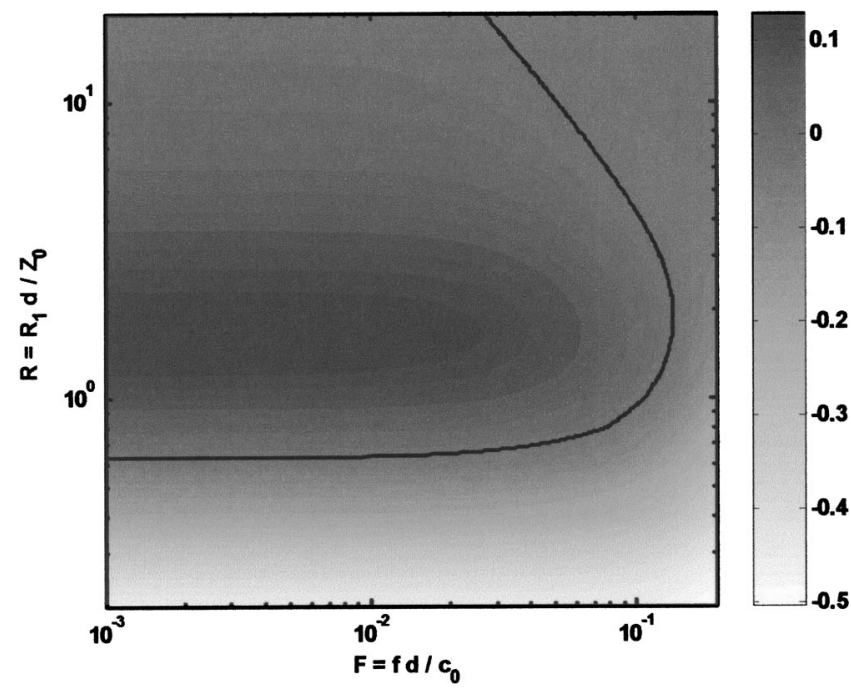

FIG. 5. Absorption difference $\left(\alpha_{\mathrm{pr}}-\alpha_{\mathrm{im}}\right)$ of the pressure-release condition against the impedance-matching condition, for a two-layer system with $D$ $=2 d$. The thick line corresponds to $\alpha_{\mathrm{pr}}=\alpha_{\mathrm{im}}$.

Therefore, the choice of the better active control condition depends on the particular noise problem to be solved. For a given absorbing material, impedance matching gives higher absorption with a thinner panel. But, the passive absorption should also be taken into account. Active absorption should be restricted to low frequency by low-pass filtering the controller. The cutoff frequency should be determined by the first peak of the passive absorption against frequency curve, which depends on the thickness of the porous layer. The thinner the porous layer, the higher the frequency that requires active control. Thus, a better choice could be a twolayer system with the flow resistance, $R_{1} d$, of the porous layer similar to the acoustic impedance of the air, and with the first peak of the passive absorption at a sufficiently low frequency. Figure 6 shows the absorption coefficient against frequency of a two-layer system with $d=4 \mathrm{~cm}, D=8 \mathrm{~cm}$, and $R_{1}=12450 \mathrm{~N} \mathrm{~s} / \mathrm{m}^{4}$, for the passive, pressure-release, and impedance-matching conditions, respectively. This is an example where active pressure-release control, low-pass filtered at $700 \mathrm{~Hz}$ combined with passive absorption offers a high-performance hybrid two-layer system.

\section{EXPERIMENTAL SETUP}

The model described above predicts higher active absorption with the pressure-release condition than with the impedance-matching condition when the passive layer has a flow resistance close to the air acoustic impedance. Beyene and Burdisso (1997) and Smith et al. (1999) reported extensive experimental evidence of the higher performance of the impedance-matching condition for low flow resistance materials. An experiment was therefore devised to measure the absorption coefficient using the pressure-release condition for comparison with the one predicted by the above model.

The experiments were carried out in a standard impedance tube with a diameter of $10 \mathrm{~cm}$ and a length of $1 \mathrm{~m}$, Fig. 7. A loudspeaker at one end generates the primary noise. The passive system, at the opposite end, consists of a porous layer in front of an air layer. The active system is comprised

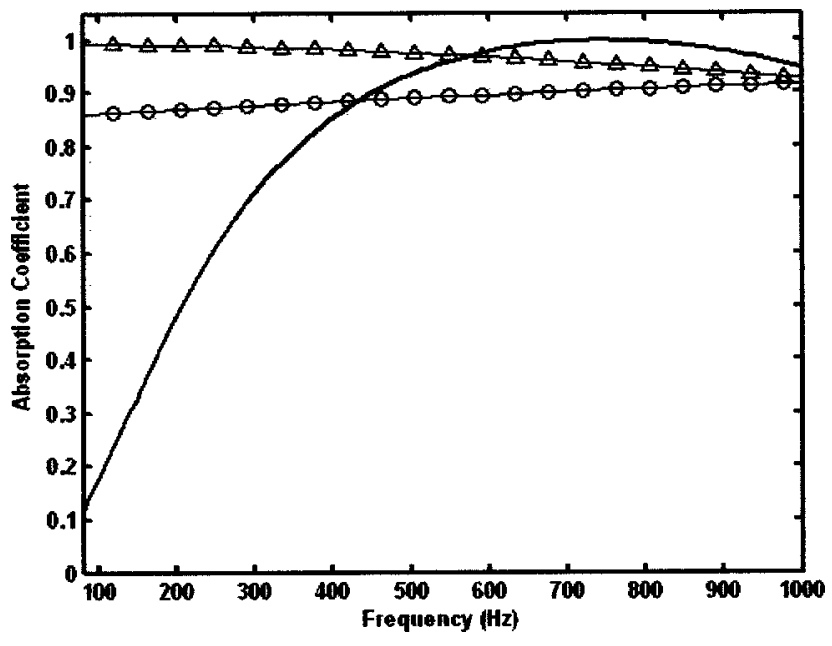

FIG. 6. Absorption coefficient against frequency of a two-layer system with $d=4 \mathrm{~cm}, \quad D=8 \mathrm{~cm}$, and $R_{1}=12450 \mathrm{~N} \mathrm{~s}^{2} \mathrm{~m}^{4}$, using passive (pressure-release $(\triangle \longrightarrow \triangle)$, and impedance-matching $(\bigcirc-\bigcirc)$ conditions.

of a loudspeaker, a microphone $\left(m_{1}\right)$, and a controller (not shown in Fig. 7). Two microphones, $m_{2}$ and $m_{3}$ (spaced either 9 or $18 \mathrm{~cm}$ apart), are used to calculate the absorption coefficient by the transfer function method. According to the corresponding international standard (ISO 10534-2), both the dimensions of the tube and the distance between the measuring microphones define an operating frequency range of 190 to $1715 \mathrm{~Hz}$, for a microphone separation of $9 \mathrm{~cm}$, and 95 to $857 \mathrm{~Hz}$, for a microphone separation of $18 \mathrm{~cm}$.

The sequence of experiments is as follows. First, a sample of absorbing material having a flow resistance close to $Z_{0}$ is chosen. Since the speed of sound and density of air are assumed to be $c_{0}=343 \mathrm{~m} / \mathrm{s}$ and $\rho_{0}=1.21 \mathrm{~kg} / \mathrm{m}^{3}$, respectively, the air acoustic impedance is $Z_{0}=415 \mathrm{~N} \mathrm{~s} / \mathrm{m}^{3}$. A layer of glass wool $3 \mathrm{~cm}$ thick with a flow resistivity of $14000 \mathrm{~N} \mathrm{~s} / \mathrm{m}^{4}$ closely fits this requirement. Then, the thickness of the air layer is selected so that the first maximum of the absorption-frequency response of the passive two-layer system coincides with the cutoff frequency of the low-pass filter of the active system, $f_{c}$. For example, for $f_{c}$ $=800 \mathrm{~Hz}$, this corresponds to a thickness $D=7 \mathrm{~cm}$. The passive absorption coefficient predicted by the model is compared with that measured in the tube. For passive measurements, a standard test sample holder (a separate unit tightly fixed to the end of the tube), replaces the secondary loudspeaker. And finally, once the passive system is optimized, the controller is configured to provide maximum active absorption.

A commercial configurable multichannel controller, using the Texas Instruments TMS320 C40 DSP, was the kernel of the active control system. It uses a feedforward strategy to produce the antinoise signal (Nelson and Elliott, 1992; Hansen and Snyder, 1997). It implements both the filtered-X LMS (adaptive FIR) and filtered-u LMS (adaptive IIR) algorithms (Elliott, 2001), and includes also a signal generator and bandpass filters for the reference, error, and control signals. The user can configure the sampling rate, the number of error inputs and control outputs (maximum eight), the type of control algorithm (filtered-x or filtered-u), the length of 


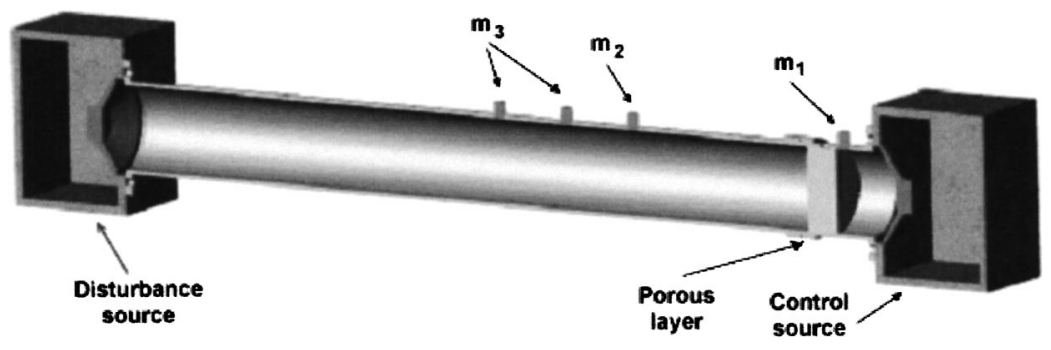

FIG. 7. Scheme of the experimental tube showing the positions of the loudspeakers and the microphones.

the control and identification filters, the convergence factor, and the low- and high-cutoff frequency of the bandpass filters. For the one-dimensional problem considered in this paper, a single channel controller was used.

\section{RESULTS AND DISCUSSION}

Several experiments were carried out using the system described above to determine the effectiveness of the pressure-release condition for the nondimensional variable $R=R_{1} d / Z_{0} \approx 1$. The passive system consists of a $3-\mathrm{cm}$ glass wool layer with $R_{1}=14000 \mathrm{~N} \mathrm{~s} / \mathrm{m}^{4}$, in front of a $7-\mathrm{cm}$ air layer. White noise was generated by the primary source at one end of the tube. First, the passive absorption curve was measured with the test sample holder at the opposite end using the signals measured by the microphones $m_{2}$ and $m_{3}$, shown in Fig. 7. Figure 8 shows that the measured passive curve matches closely with the theoretical one predicted by the model, except for small differences (less than 0.05) between 100 and $300 \mathrm{~Hz}$. The passive system affords high absorption above $800 \mathrm{~Hz}$; thus, the active system should be configured to perform below this frequency.

Next, the passive test sample holder was replaced by the secondary loudspeaker, and the active controller was optimized for maximum absorption. The same white-noise signal used to drive the primary loudspeaker was also utilized as reference signal for the controller. Microphone $m_{1}$ was positioned in the air cavity $0.5 \mathrm{~cm}$ from the absorbing layer to provide the error signal. Both the reference and the error

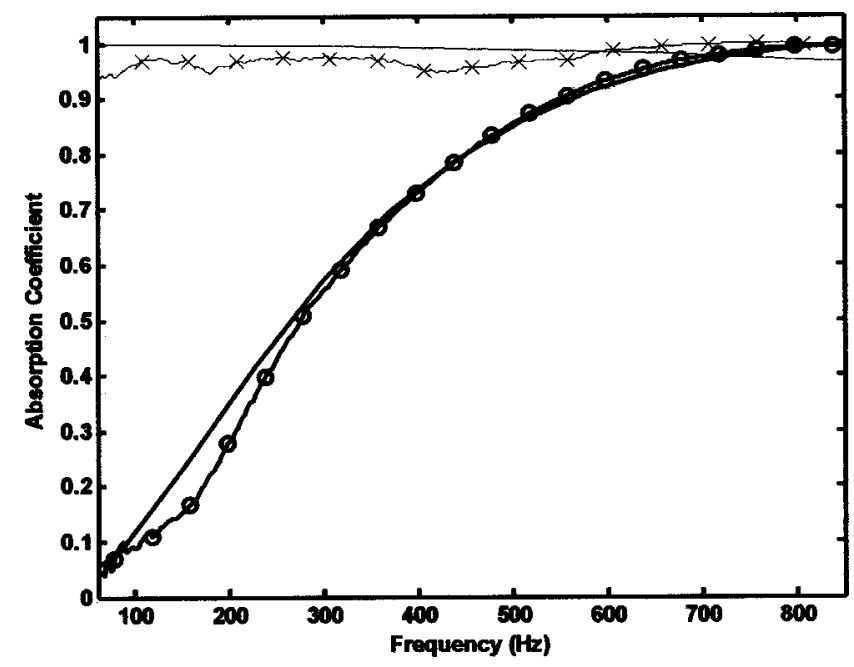

FIG. 8. Absorption coefficient against frequency of a passive-active twolayer system using the pressure-release condition: (— - ) passive theoretical, $(\bigcirc-\bigcirc)$ passive experimental, $(-)$ active theoretical, and $(\times-\times)$ active experimental. inputs were low-pass filtered at $800 \mathrm{~Hz}$. The sampling rate was set at $4 \mathrm{kHz}$. Due to the broadband nature of the disturbance, IIR filters were used for both the control and identification adaptive algorithms of the controller. In this context, optimizing the performance of the controller means finding the best number of forward and backward filter taps, and the best convergence factors that minimize the error signal. Once the controller was optimized and maximum cancellation was obtained, the absorption coefficient was measured again using the two microphones $m_{2}$ and $m_{3}$ spaced $18 \mathrm{~cm}$ apart. Figure 8 shows excellent agreement between the absorption coefficient predicted by the model and that measured in the experiment. The active system yields absorption values above 0.95 in the range $90-850 \mathrm{~Hz}$.

Additional experiments with other absorbing materials (for example, melamine foam) gave similar results. Therefore, these results demonstrate the feasibility of designing hybrid passive-active systems with high absorption using the pressure-release condition. Moreover, from an electronic hardware point of view, it is much simpler to implement than the impedance-matching condition.

\section{CONCLUSIONS}

A simple two-layer hybrid passive-active model for plane waves considering both the geometrical and the material characteristics of the passive system, allows a better comparison between the pressure-release and the impedancematching control conditions. While the impedance-matching condition requires additional electronic hardware to decompose the standing waves into forward and backward planewave components, the pressure-release condition needs to drive the pressure measured to zero using only one microphone. Moreover, the pressure-release condition affords higher absorption when the flow resistance of the porous layer is similar to the acoustic impedance of air. Experimental data measured in a standard impedance tube under the pressure-release condition validate this prediction. The absorption coefficient afforded by a $3-\mathrm{cm}$ glass wool layer spaced $7 \mathrm{~cm}$ from the secondary loudspeaker is higher than 0.95 at frequencies between 90 and $850 \mathrm{~Hz}$. The model predicts better performance of the impedance-matching condition for absorbing materials with flow resistance less than half of the acoustic impedance of air.

\section{ACKNOWLEDGMENTS}

The authors are grateful to the Spanish Ministry of Science and Technology for funding this research under Grant No. DPI2001-1613-C02-01. We acknowledge fruitful discussions with Dr. David K. Anthony. 
Allard, J. F., and Champoux, Y. (1992). "New empirical equations for sound propagation in rigid frame fibrous materials," J. Acoust. Soc. Am. 91, 3346-3353.

Beyene, S., and Burdisso, R. A. (1997). "A new hybrid passive/active noise absorption system," J. Acoust. Soc. Am. 101, 1512-1515.

Delany, M. E., and Bazley, E. N. (1970). "Acoustical properties of fibrous absorbent materials," Appl. Acoust. 3, 105-116.

Elliott, S. J. (2001). Signal Processing for Active Control (Academic, London), pp. 132-166.

Furstoss, M., Thenail, D., and Galland, M. A. (1997). "Surface impedance control for sound absorption: Direct and hybrid passive/active strategies," J. Sound Vib. 203, 219-236.

Guicking, D., Karcher, K., and Rollwage, M. (1985). "Coherent active methods for application in room acoustics," J. Acoust. Soc. Am. 78, $1426-1434$.
Guicking, D. (1990). "On the invention of active noise control by Paul Lueg," J. Acoust. Soc. Am. 87, 2251-2254.

Hansen, C. H., and Snyder, S. D. (1997). Active Control of Noise and Vibration (Spon, London), pp. 559-576.

Mechel, F. P. (1988). "Design charts for sound absorber layers," J. Acoust. Soc. Am. 83, 1002-1013.

Nelson, P. A., and Elliott, S. J. (1992). Active Control of Sound (Academic, London), pp. 161-179.

Orduña-Bustamante, F., and Nelson, P. A. (1992). "An adaptive controller for the active absorption of sound," J. Acoust. Soc. Am. 91, 27402747.

Smith, J. P., Johnson, B. D., and Burdisso, R. A. (1999). "A broadband passive-active sound absorption system,” J. Acoust. Soc. Am. 106, 26462652. 